\title{
Short infrared wavelength quantum cascade detectors based on m-plane $\mathrm{ZnO} / \mathrm{ZnMgO}$ quantum wells.
}

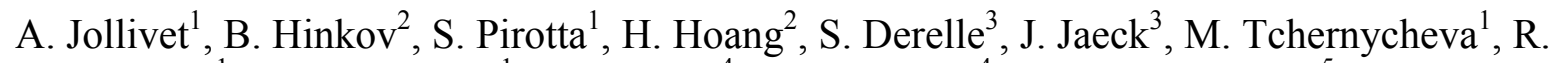
Colombelli $^{1}$, A. Bousseksou ${ }^{1}$, M. Hugues ${ }^{4}$, N. Le Biavan ${ }^{4}$, J. Tamayo-Arriola ${ }^{5}$, M. Montes Bajo $^{5}$, L. Rigutti ${ }^{6}$, A. Hierro ${ }^{5}$, G. Strasser ${ }^{2}$, J-M. Chauveau ${ }^{4}$, and F. H. Julien ${ }^{1}$

${ }^{1}$ Centre de Nanosciences et de Nanotechnologies (C2N), CNRS UMR 9001, Univ. Paris-Sud, Université Paris-Saclay, 91120 Palaiseau, France.

${ }^{2}$ TU-Wien, Nanocenter Campus-Gußhaus, Gußhausstraße 25, Gebäude-CH, A-1040 Vienna, Austria.

${ }^{3}$ ONERA/DOTA/CIO, Chemin de la Hunière, 91761 Palaiseau Cedex, France.

${ }^{4}$ Université Côte d'Azur, CNRS, CRHEA, 06560 Valbonne, France.

${ }^{5}$ ISOM, Universidad Politécnica de Madrid, 28040 Madrid, Spain.

${ }^{6}$ Normandie Univ, UNIROUEN, INSA Rouen, CNRS, Grp Phys Mat, F-76000 Rouen, France.

Corresponding author: francois.julien@c2n.upsaclay.fr

\begin{abstract}
:
This paper reports on the demonstration of QCDs based on $\mathrm{ZnO} / \mathrm{ZnMgO}$ QWs grown by molecular beam epitaxy on an m-plane $\mathrm{ZnO}$ substrate. The TM-polarized intersubband absorption is peaked at $3 \mu \mathrm{m}$ wavelength. The sample has been processed in the form of square mesas with sizes ranging from $10 \times 10 \mu \mathrm{m}^{2}$ up to $100 \mathrm{x} 100 \mu \mathrm{m}^{2}$. The $\mathrm{I}-\mathrm{V}$ characteristics reveal that $86 \%$ of the 260 devices are operational and that the surface leakage current is negligible at room temperature, which is not the case at $77 \mathrm{~K}$. The photocurrent spectroscopy of $100 \times 100 \mu \mathrm{m}^{2}$ QCDs reveals a photocurrent resonance at $2.8 \mu \mathrm{m}$ wavelength, i.e. slightly blue-shifted with respect to the intersubband absorption peak. The photocurrent persists up to room temperature. The calibrated peak responsivity amounts to $0.15 \mathrm{~mA} / \mathrm{W}$ under irradiation at Brewster's angle of incidence. This value allows to estimate the transfer efficiency $(1.15 \%)$ of the photoexcited electrons into the active QW of the next period.
\end{abstract}


Quantum cascade detectors (QCD) are semiconductor photovoltaic devices operated at zero bias. ${ }^{1,2}$ With respect to quantum well infrared (QWIPs) photoconductors, the absence of dark current in QCDs is particularly favorable to enhance the signal-to-noise ratio. One stage of the multi-period active region of a QCD device consists of an active quantum well (QW), where an intersubband (ISB) absorption takes place, and an extractor stage designed to transfer the photoexcited electrons from the active QW to the next period active QW. This charge transfer gives rise to a photovoltage or a photocurrent if the device is connected to a load resistor. The design of the extractor stage usually relies on multiple QWs to achieve a staircase of bound electronic states separated by the energy of one longitudinal optical (LO) phonon. Indeed, fast electron LO-phonon scattering provides efficient electron transfer. So far, QCDs have been demonstrated in the arsenide, antimonide, III-nitride and $\mathrm{ZnCdSe} / \mathrm{ZnCdMgSe}$ material systems in the wavelength range from 1 to $84 \mu \mathrm{m} .{ }^{1-11}$ A further appealing feature of QCDs is their fast intrinsic response. ${ }^{12}$ For instance, GaN-based QCDs operating at $1.5 \mu \mathrm{m}$ wavelength have revealed a $-3 \mathrm{~dB}$ frequency bandwidth exceeding $40 \mathrm{GHz} .{ }^{13}$

Wurzite $\mathrm{ZnO} / \mathrm{ZnMgO}$ is considered as a new material for ISB devices. ISB absorption has been recently observed in both c-plane and m-plane crystalline structures, the latter favoring the design of QCD devices because of the absence of internal electric fields. ${ }^{14-16}$ Similarly to III-nitride semiconductors, one main advantage for QCD operation is the large energy of LOphonons in $\mathrm{ZnO}(72 \mathrm{meV})$, which provides room for high detectivity devices. Indeed, the detectivity is proportional to the square root of the resistance at zero bias $\left(\mathrm{R}_{0}\right)$ times the area of the detector (A). ${ }^{1} \mathrm{R}_{0}$ in a photovoltaic device like the QCD is limited by the non-resonant electron tunneling from the ground state of the active QW to one of the extractor states assisted by the absorption of LO-phonons. ${ }^{17}$ In highly polar materials like GaN or $\mathrm{ZnO}$, the Frölich interaction between electrons and LO-phonons is strongly enhanced leading to an enhanced electronic transfer efficiency within the extractor. However, no QCD based on ZnO heterostructures has been reported so far.

In this paper, we report on the demonstration of QCDs based on $\mathrm{ZnO} / \mathrm{ZnMgO}$ QWs grown by molecular beam epitaxy (MBE) on an m-plane $\mathrm{ZnO}$ substrate. Transmission spectroscopy using a Fourier Transform Infrared (FTIR) spectrometer reveals a TM-polarized absorption peaked at $3 \mu \mathrm{m}$ wavelength attributed to the ISB absorption in the active QWs. The sample has been processed in the form of 260 square mesas with a size ranging from $10 \times 10 \mu \mathrm{m}^{2}$ up to $100 \times 100 \mu \mathrm{m}^{2}$, with special emphasis on the sidewall passivation. The I-V characteristics reveal that $86 \%$ of the devices are operational. The current density versus voltage is independent of the mesa size at room temperature, indicating that no surface leakage currents are present. However, this is not the case at $77 \mathrm{~K}$, revealing that the electron leakage through the mesa sidewalls dominates the transport at low temperatures. The photocurrent spectroscopy has been performed by illuminating the top surface of the $100 \times 100 \mu \mathrm{m}^{2}$ QCDs at Brewster's angle of incidence. It exhibits a photocurrent resonance at $2.8 \mu \mathrm{m}$ wavelength, i.e. slightly blue-shifted with respect to the ISB absorption peak. The photocurrent persists up to room temperature. The calibrated peak responsivity at $77 \mathrm{~K}$ amounts to $0.15 \mathrm{~mA} / \mathrm{W}$ under irradiation at Brewster's angle of incidence. This value allows to estimate the transfer efficiency of the photoexcited electrons into the active QW of the next period. 
Figure 1 shows the conduction band profile of the originally designed sample calculated by solving the Schrödinger equation using the effective mass approximation. The layer thickness sequence for one period starting from the active QW is 2.6/1.5/0.9/1.5/1.2/1.3/1.7/1.2 where underlined figures correspond to $\mathrm{ZnMgO}$ barriers with a $40 \% \mathrm{Mg}$ concentration. The target wavelength of this design is $3.7 \mu \mathrm{m}$. As seen in Fig. 1, the purpose of this design is to provide a significant envelope function overlap of the excited state of the active QW with the first extractor QW state in order to ensure a fast electron transfer within the extractor mediated by the electron-LO phonon scattering. However, as discussed below the doping of the active QW leads to band bending effects, which perturbs the alignment of the states.

The sample was grown on $10 \times 10 \mathrm{~mm}$ m-oriented $\mathrm{ZnO}$ substrates (Crystec). Prior to growth, the substrates were annealed at $1065{ }^{\circ} \mathrm{C}$ under an oxygen (O) atmosphere to reveal atomic steps. The layer sequence was then grown in a MBE system (RIBER, Epineat) equipped with zinc $(\mathrm{Zn})$ and magnesium $(\mathrm{Mg})$ cells and one gallium $(\mathrm{Ga})$ cell for $\mathrm{n}$-type doping. The atomic oxygen was provided by a plasma cell operating at $420 \mathrm{~W}$ with an oxygen-flow of $0.3 \mathrm{sccm}$. The substrate temperature was set to $420{ }^{\circ} \mathrm{C}$ during growth. The $\mathrm{Zn}$ and $\mathrm{O}$ fluxes were adjusted to be close to stoichiometry to limit defect concentrations and resulted in a growth rate of $170 \mathrm{~nm} / \mathrm{h}$. The $2.6 \mathrm{~nm}$ thick active QWs were $\mathrm{n}$-doped with Ga at a concentration of $2 \times 10^{19} \mathrm{~cm}^{-3}$. The 20 period active region was grown on top of a $1 \mu \mathrm{m}$ thick $\mathrm{ZnO}$ bottom contact layer doped with $\mathrm{Ga}$ at $2 \times 10^{19} \mathrm{~cm}^{-3}$ and was capped with a $100 \mathrm{~nm}$ thick top $\mathrm{ZnO}$ contact layer Ga-doped at the same concentration. Optical inspection reveals no cracks in the sample after growth. Similar samples with $\mathrm{ZnO} / \mathrm{Zn}_{0.6} \mathrm{Mg}_{0.4} \mathrm{O}$ superlattices were investigated by scanning transmission electron microscopy (STEM) and atomic probe tomography (APT). 18 The results show that the interfaces are abrupt and that the actual thickness of the $\mathrm{ZnO}$ QWs is within \pm 1 monolayer (ML, $0.23 \mathrm{~nm}$ ) close to the nominal thickness. However, due to the different adatom diffusion during growth along the $c$ - and $a$-axis in the plane of the layers, a spatially coherent corrugation develops starting from the bottom contact layer up to the cap layer. ${ }^{18}$ In addition, APT reveals that the Mg concentration in the barriers varies by $\pm 10 \%$ in absolute values at the bottom and the top of the corrugations.

The infrared transmission of the sample was probed in a multi-pass waveguide configuration with two internal reflections using a Bruker Vertex 70 FTIR spectrometer. Figure 2 shows the TM-polarized transmission divided by the TE transmission at $77 \mathrm{~K}$ and $300 \mathrm{~K}$. The TMpolarized ISB absorption is peaked at $416 \mathrm{meV}(2.98 \mu \mathrm{m}$ wavelength) with a full width at half maximum (FWHM) of $112 \mathrm{meV}$ at $77 \mathrm{~K}$. The absorbance per QW is on the order of $5.8 \times 10^{-3}$. This rather small value is a consequence of the large effective mass in $\mathrm{ZnO}$, which translates into a lower oscillator strength when compared to semiconductors like GaAs. Note that the peak absorption is blue-shifted with respect to the design target wavelength. This is a consequence of the depolarization shift, by which the oscillating electrons screen the electric field, blue-shifting the effective absorption energy. This effect is quite large for a high doping, like the one used in this structure, and especially for $\mathrm{ZnO}$ due to the very low value of its background dielectric constant (3.69). ${ }^{19}$ Simulations accounting for this effect together with Poisson and exchange correlation, and using the experimentally observed broadening of the transition, predict a peak absorption around $3 \mu \mathrm{m}$ wavelength (see the dotted curve in Fig. 2), 
which is very close to the experimental value. Details of the calculations can be found in Ref. ${ }^{19}$. Based on the calculated ISB dipole length $(0.6 \mathrm{~nm})$ and the experimental FWHM, the electron density in the active QW is estimated to be $4.6 \times 10^{12} \mathrm{~cm}^{-2}$, which corresponds to a $1.8 \times 10^{19} \mathrm{~cm}^{-3}$ volume concentration close to the nominal doping concentration. The Fermi energy with respect to the ground state is deduced to be $45.7 \mathrm{meV}$ at $77 \mathrm{~K}$.

The sample was processed as 260 square mesas with a size of $10 \times 10,20 \times 20,30 \times 30,50 \times 50$, $75 \times 75$ and $100 \times 100 \mu \mathrm{m}^{2}$. The metallization of the top contact is restricted to the perimeter of the mesa to allow topside-irradiation at Brewster angle of incidence. In order to suppress surface leakage currents, the devices were treated with $\mathrm{H}_{2} \mathrm{O}_{2}$ at $95^{\circ} \mathrm{C}$ after etching of the mesas in a $\mathrm{CH}_{4}$-based RIE-plasma. ${ }^{20}$

The I-V characteristics reveal that $86 \%$ of all mesa devices are operational. Figure 3 a) shows the I-V characteristics at room temperature of 17 QCDs with a size of $100 \times 100 \mu \mathrm{m}^{2}$. The characteristics exhibit an asymmetric diode behavior as expected from the asymmetry of the conduction band profile.

As seen in Fig. 3 a), there is only a little dispersion between the I-V curves of the various QCDs. $\mathrm{R}_{0}$ is in the range of $1.42-1.52 \mathrm{k} \Omega$ at $300 \mathrm{~K}$ for these $100 \times 100 \mu \mathrm{m}^{2}$ QCDs. Figure $3 \mathrm{~b}$ ) presents the current density versus voltage $(\mathrm{J}-\mathrm{V})$ characteristics at $300 \mathrm{~K}$ for all sizes of QCDs. The absence of dispersion between the curves is a clear indication that the current is proportional to the surface and consequently that the sidewall leakage is negligible at room temperature. This is not the case at $77 \mathrm{~K}$. The inset of Fig. $3 \mathrm{~b}$ ) shows the I-V characteristics divided by the mesa perimeter at $77 \mathrm{~K}$ for all mesa sizes. As seen, the characteristics at $77 \mathrm{~K}$ for all mesa sizes are well superimposed which is a signature that the current is dominated by sidewall leakages. In addition, $\mathrm{R}_{0}$ is found to be inversely proportional to the mesa perimeter and not to the mesa surface. The $R_{0}$ value increases from $71.7 \mathrm{k} \Omega(1.52 \mathrm{k} \Omega)$ at $300 \mathrm{~K}$ to 1.75 $\mathrm{M} \Omega(0.18 \mathrm{M} \Omega)$ at $77 \mathrm{~K}$ for $10 \mathrm{x} 10 \mu \mathrm{m}^{2}\left(100 \times 100 \mu \mathrm{m}^{2}\right)$ mesas, respectively. Figure $\left.3 \mathrm{c}\right)$ shows an Arrhenius plot of $\mathrm{R}_{0} \mathrm{~A}$. The activation energy at $300 \mathrm{~K}$ is around $180 \mathrm{meV}$ and decreases rapidly at lower temperatures due to the onset of current facet leakages. This value of the activation energy at $300 \mathrm{~K}$ is close to the energy difference between the ground state of the first extractor and the ground state of the active QW in agreement with Ref ${ }^{17}$.

Photocurrent (PC) spectroscopy was performed on the 100x100 $\mu \mathrm{m}^{2}$ mesas QCDs using the FTIR spectrometer glow-bar source, that was mechanically chopped and a lock-in amplifier. The light impinging on the QCDs was focused with a $10 \mathrm{~cm}$ focal length parabolic mirror at Brewster's angle of incidence $\left(62^{\circ}\right)$. Figure 4 shows the photocurrent spectra for TM- and TEpolarized light at $77 \mathrm{~K}$. The photocurrent spectrum is strongly TM-polarized as expected from detectors based on ISB absorption. The residual TE-polarized signal may arise from diffraction effects on the facets of the mesas. The PC spectra are peaked at $2.8 \mu \mathrm{m}$ wavelength (443 meV) with a FWHM of $97 \mathrm{meV}$. The PC spectrum is slightly blue-shifted with respect to the absorption spectra. The narrower linewidth and the spectral shift suggest that the electron transfer between the active QWs and the extractor region may be favored in the plane of the layers in barrier regions containing a higher $\mathrm{Mg}$ content. 
The responsivity of a typical device was calibrated under a mechanically chopped illumination by a $1200^{\circ} \mathrm{C}$ black-body source and a narrow band-pass filter centered at $2.7 \mu \mathrm{m}$ wavelength, i.e. close to the PC peak wavelength. The results corrected for the filter and cryostat window transmissions as well as the spot size onto the detector reveal a peak responsivity for TM-polarized light on the order of $0.15 \mathrm{~mA} / \mathrm{W}$ under illumination of the top surface at Brewster's angle of incidence. Note that this value could be strongly enhanced by both increasing the doping of the active QWs and making use of a grating or a $45^{\circ}$ wedge configuration. The response could also be improved by more than two orders of magnitude using a waveguide configuration for a perfect TM coupling and complete absorption. ${ }^{13}$ Based on the responsivity, one can estimate the electron transfer efficiency from period to period, $\eta$, defined as the ratio of the photocurrent to the sum of the photocurrent and the parasitic current. The latter arises from electrons in the excited state relaxing back to the ground state of the same active QW. Based on simple rate equations and assuming a negligible depletion of the incoming light intensity within the 20 periods which is the case in the present study, one can show that:

$$
\eta=E_{12} R /(a e)
$$

where $E_{12}$ is the ISB transition energy, $R$ is the responsivity, $a \sim 5.8 \times 10^{-3}$ is the absorbance per QW and $e$ is the electron charge. The transfer efficiency is found to be of the order of $\sim 1.15 \%$. This value is rather low with respect to the best results published so far. ${ }^{10}$ This low value may be attributed to the $\mathrm{Mg}$ content fluctuations in the barrier layers revealed by APT measurements as well as the band bending induced by the doping of the active well. Figure 5 shows the conduction band profile of one period of the QCD obtained by self-consistently solving the Schrödinger and Poisson equations for two Mg concentrations of $30 \%$ and $50 \%$, respectively. As seen in Fig. 5 a), the energy spacing between the excited state of the active QW and the first extractor QW state is around $91 \mathrm{meV}$ while for a $50 \% \mathrm{Mg}$ content (Fig. $5 \mathrm{~b}$ ) it is resonant with the LO-phonon energy $(72 \mathrm{meV})$. Assuming as a first approximation bulklike phonons, the electron-LO phonon scattering time scales as $|\mathrm{q}|^{2}$, where $\mathrm{q}$ is the momentum of the phonon. As a consequence, the scattering time is expected to be longer when the energy spacing is larger than the LO-phonon energy, which is the case for the $30 \% \mathrm{Mg}$ content QWs. Assuming a more refined 2D phonon model, it was shown that for a resonant LO-phonon transition the scattering is dominated by interface phonon modes leading to very shot scattering times. ${ }^{21}$ As a result, the electron transfer efficiency into the extractor is expected to be much favored for the higher $\mathrm{Mg}$ content structure. In addition, the $30 \% \mathrm{Mg}$ content design exhibits significant parasitic electron scattering channels towards the ground state of the same period active QW ground state (see dashed lines in Fig.5 a) which reduces the electron transfer efficiency towards the next period and the responsivity. Both effects may explain the blue-shift of the photoresponse with respect to the absorption measurements. The $\mathrm{Mg}$ fluctuation appears as the main factor leading to a rather low responsivity.

Finally, separate experiments under illumination by the blackbody source reveal that the photoresponse persists up to room temperature. However the Johnson noise measured in the 
dark with a $1 \mathrm{sec}$ integration time increases exponentially with the temperature and becomes comparable to the photocurrent signal at $320 \mathrm{~K}$.

In conclusion, we have demonstrated QCDs based on $\mathrm{ZnO} / \mathrm{ZnMgO}$ QWs grown by molecular beam epitaxy (MBE) on an m-plane $\mathrm{ZnO}$ substrate. The ISB absorption in the active QWs is peaked at $3 \mu \mathrm{m}$ wavelength, blue-shifted from the target wavelength of $3.7 \mu \mathrm{m}$ because of the depolarization shift. The sample has been processed in the form of 260 square mesas with various mesa sizes. The I-V characteristics reveal that $86 \%$ of the devices are operational and that the surface leakage current is negligible at room temperature, which is not the case at 77 $\mathrm{K}$. The photocurrent spectroscopy reveals a photocurrent resonance at $2.8 \mu \mathrm{m}$ wavelength, i.e. slightly blue-shifted with respect to the ISB absorption peak. The photocurrent persists up to room temperature. The calibrated peak responsivity amounts to $0.15 \mathrm{~mA} / \mathrm{W}$ under irradiation at Brewster's angle of incidence. The transfer efficiency of electrons between successive periods is estimated to be around $1.15 \%$ and could be enhanced by optimizing the design and growth of the QCD structure.

Acknowledgments: This work was funded by the European Union's Horizon 2020 Research and Innovation FET-Open Program under Grant Agreement No. 665107 (project ZOTERAC). 


\section{References:}

${ }^{1}$ L. Gendron, M. Carras, A. Huynh, V. Ortiz, C. Koeniguer, and V. Berger, Appl. Phys. Lett. 85,2824 (2004).

${ }^{2}$ M. Graf, G. Scalari, D. Hoffstetter, J. Faist, H. Beere, E. Lindfield, D. Ritchie, and G. Davies, Appl. Phys. Lett. 84, 475 (2004).

${ }^{3}$ F. R. Giorgetta, E. Baumann, D. Hofstetter, C. Manz, Q. Yang, K. K€ohler, and M. Graf, Appl. Phys. Lett. 91, 111115 (2007).

${ }^{4}$ F. R. Giorgetta, E. Baumann, M. Graf, Q. K. Yang, C. Manz, K. Kohler, H. E. Beere, D. A. Ritchie, E. Linfield, A. G. Davies, Y. Fedoryshyn, H. Jackel, M. Fischer, J. Faist, and D. Hofstetter, IEEE J. Quantum Electron. 45, 1029-1042 (2009), and reference therein.

${ }^{5}$ A. Buffaz, M. Carras, L. Doyennette, A. Nedelcu, X. Marcadet, and V. Berger, Appl. Phys. Lett. 96, 172101 (2010).

${ }^{6}$ A. Vardi, G. Bahir, F. Guillot, C. Bougerol, E. Monroy, S. E. Schacham, M. Tchernycheva, and F. H. Julien, Appl. Phys. Lett. 92, 011112 (2008).

${ }^{7}$ A. Vardi, N. Kheirodin, L. Nevou, H. Machhadani, L. Vivien, P. Crozat, M. Tchernycheva, R. Colombelli, F. H. Julien, F. Guillot, C. Bougerol, E. Monroy, S. Schacham, and G. Bahir, Appl. Phys. Lett. 93, 193509 (2008).

${ }^{8}$ S. Sakr, Y. Katsov, S. Haddadi, M. Tchernycheva, L. Vivien, I. Sarigianni- dou, N. Isac, E. Monroy, and F. H. Julien, Electron. Lett. 46, 1685 (2010).

${ }^{9}$ S. Sakr, E. Giraud, A. Dussaigne, M. Tchernycheva, N. Grandjean, and F. H. Julien, Appl. Phys. Lett. 100, 181103 (2012).

${ }^{10}$ S. Sakr, E. Giraud, M. Tchernycheva, N. Isac, P. Quach, E. Warde, N. Grandjean, and F. H. Julien, Appl. Phys. Lett. 101, 251101 (2012).

${ }^{11}$ A. P. Ravikumar, J. De Jesus, M. C. Tamargo, C. F. Gmachl, Appl. Phys. Lett. 107, 141105 (2015).

${ }^{12}$ A. Vardi, S. Sakr, J. Mangeney, K. W. Kandaswamy, E. Monroy, M. Tchernycheva, S. E. Schacham, F. H. Julien, and G. Bahir, Appl. Phys. Lett. 99, 202111 (2011).

${ }^{13}$ S. Sakr, P. Crozat, D. Gacemi, Y. Kotsar, A. Pesach, P. Quach, N. Isac, M. Tchernycheva, L. Vivien, G. Bahir, E. Monroy, F. H. Julien, Appl. Phys. Lett. 102, 011135 (2013).

${ }^{14}$ M. Belmoubarik, K Ohtani, and H Ohno, Appl. Phys. Lett. 92, 191906 (2008).

${ }^{15}$ K. Zhao, G. Chen, B. S. Li, and A. Shen, Appl. Phys. Lett.. 104, 212104 (2014).

${ }^{16}$ N. Le Biavan, M. Hugues, M. Montes Bajo, J. Tamayo- Arriola, A. Jollivet, D. Lefebvre, Y. 
Cordier, B. Vinter, F.-H. Julien, A. Hierro, and J.-M. Chauveau, Appl. Phys. Lett. 111, 231903 (2017).

${ }^{17}$ A. Gomez, N. Péré-Laperne, L.-A. de Vaulchier, C. Koeniguer, A. Vasanelli, A. Nedelcu, X. Marcadet, Y. Guldner, and Vincent Berger, Phys. Rev. B 77, 085307 (2008)

${ }^{18}$ E. Di Russo, L. Mancini, F. Moyon, S. Moldovan, J. Houard, F.H. Julien, M. Tchernycheva, J.-M. Chauveau, M. Hugues, G. Da Costa, I. Blum, W. Lefebvre, D. Blavette, L. Rigutti, Appl. Phys. Lett. 111, 032108 (2017).

${ }^{19}$ M. Montes Bajo, J. Tamayo-Arriola, M. Hugues, J. M. Ulloa, N. Le Biavan, R. Peretti, F. H. Julien, J. Faist, J.-M. Chauveau, and A. Hierro, Phys. Rev. Appl. 10, 024005 (2018).

${ }^{20}$ S.-W. Na, M. H. Shin, Y. M. Chung, J. G. Han, and N.-E. Lee, Journ. of Vacuum Science \& Technology A, 23, 898 (2005).

${ }^{21}$ J.L. Educato, J.-P. Leburton, P. Boucaud, P. Vagos, F.H. Julien, Phys. Rev. B, 47, 1294912952 (1993). 


\section{Figure caption:}

Figure 1: Conduction band profile of the $\mathrm{ZnO}-\mathrm{ZnMgO}$ QCD. The red arrows denote the ISB absorption in the active QWs and the green arrows the relaxation paths via electron-LO phonon interaction.

Figure 2: Ratio of the transmission spectra of the sample for TM and TE polarized light at 77 (green curve) and $300 \mathrm{~K}$ (red curve). The dotted line shows the simulated spectrum.

Figure 3: (a) Room temperature I-V characteristics of 17 devices with a mesa size of 100x100 $\mu \mathrm{m}^{2}$. The inset shows a top-view optical image of one of the mesas; (b) RT J-V characteristics of QCDs with a mesa size ranging from $10 \times 10 \mu \mathrm{m}^{2}$ to $100 \times 100 \mu \mathrm{m}^{2}$. The inset shows the J-V characteristics at $77 \mathrm{~K}$. (c) Arrhenius plot of $\mathrm{R}_{0} \mathrm{~A}$ versus the inverse temperature. The dots are experimental data. The curve is a polynomial fit. The dashed line shows the activation energy fit at $300 \mathrm{~K}$.

Figure 4: Photocurrent spectra at $77 \mathrm{~K}$ for TM- and TE-polarized light of a $100 \times 100 \mu \mathrm{m}^{22}$ QCD. The dip at $400 \mathrm{meV}$ is due to $\mathrm{CO}_{2}$ absorption.

Figure 5: Conduction band profile of the doped QCDs for a $\mathrm{Mg}$ content in the barriers of 30\% (a) and 50\% (b). The dashed arrows show some parasitic electron transfer channels. 


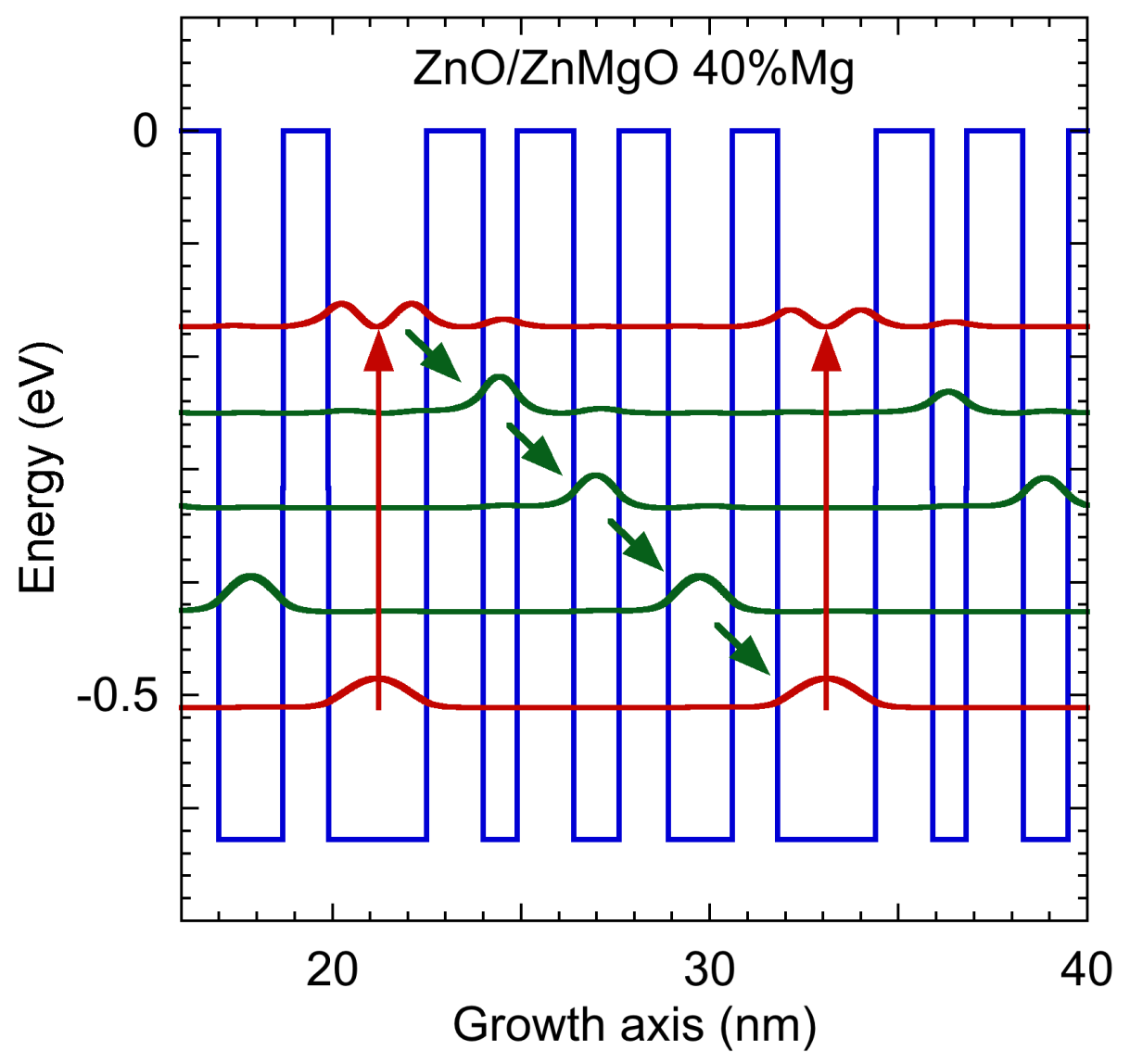




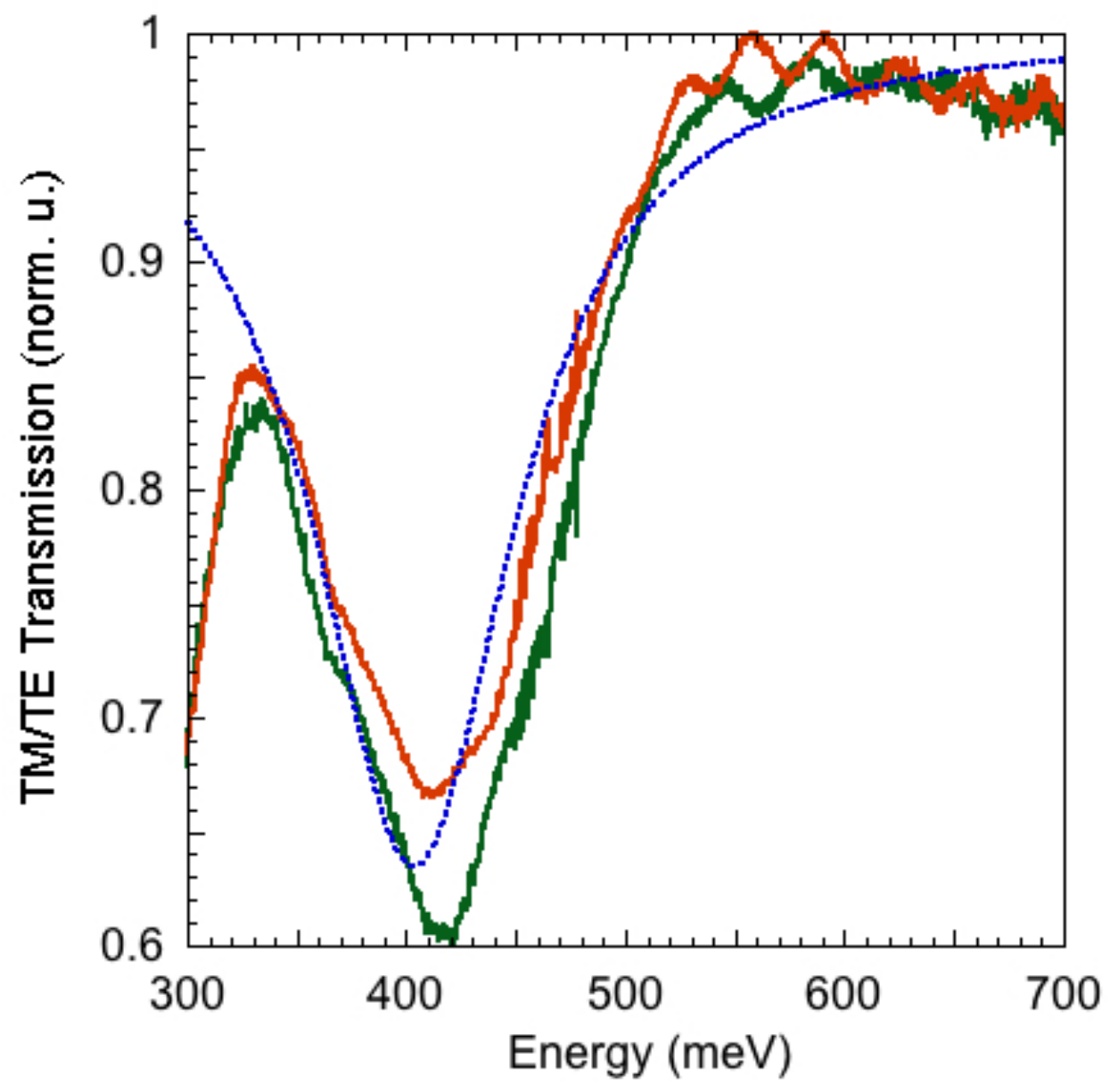



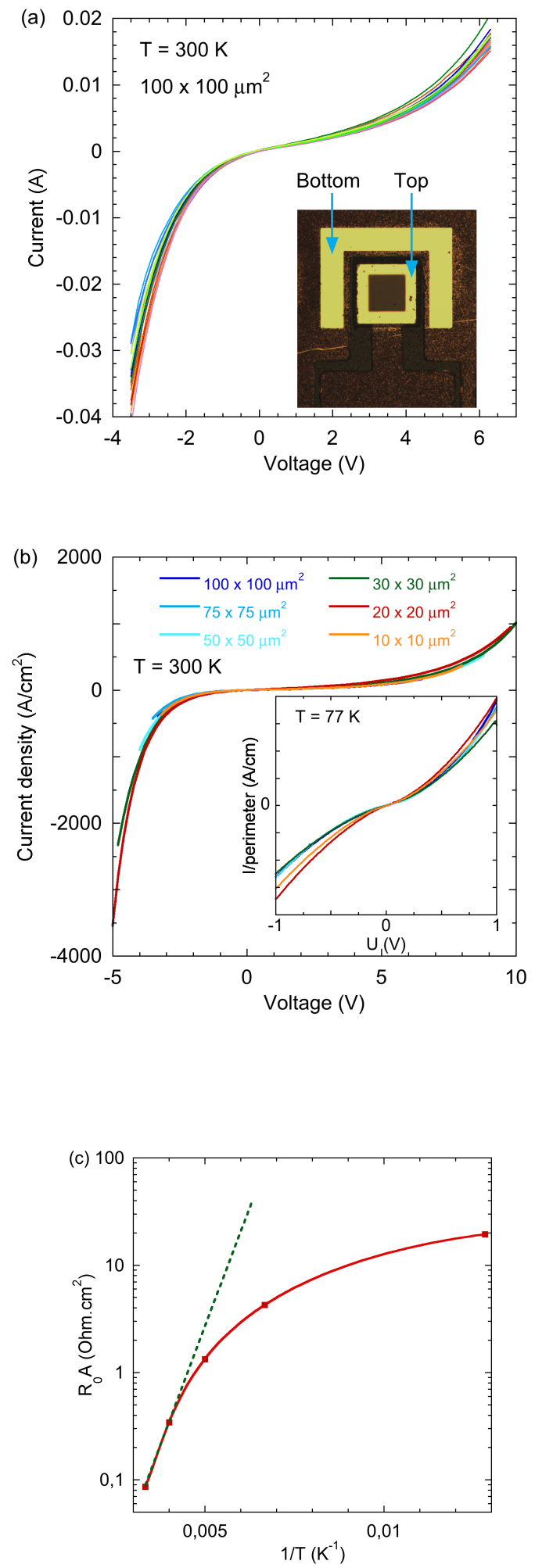


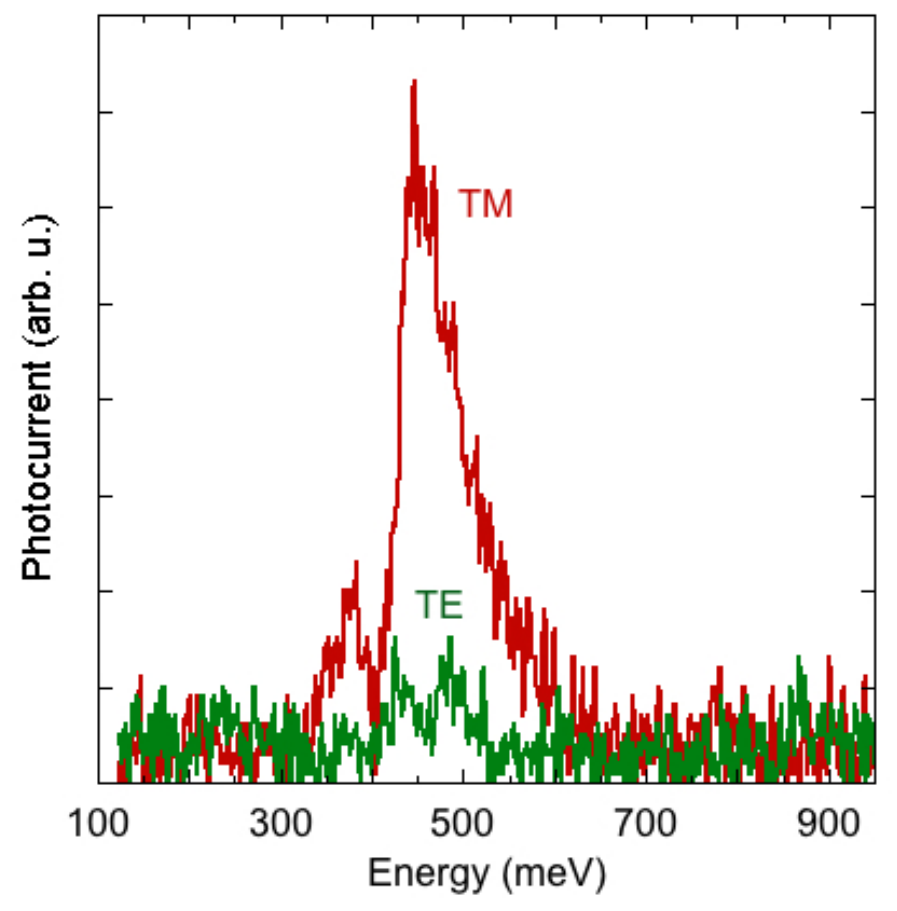


(a) 0.4

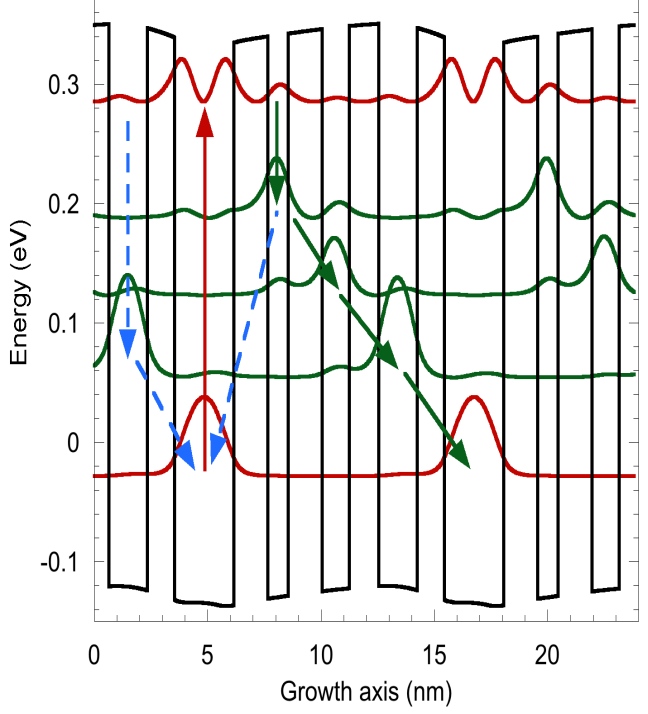

(b)

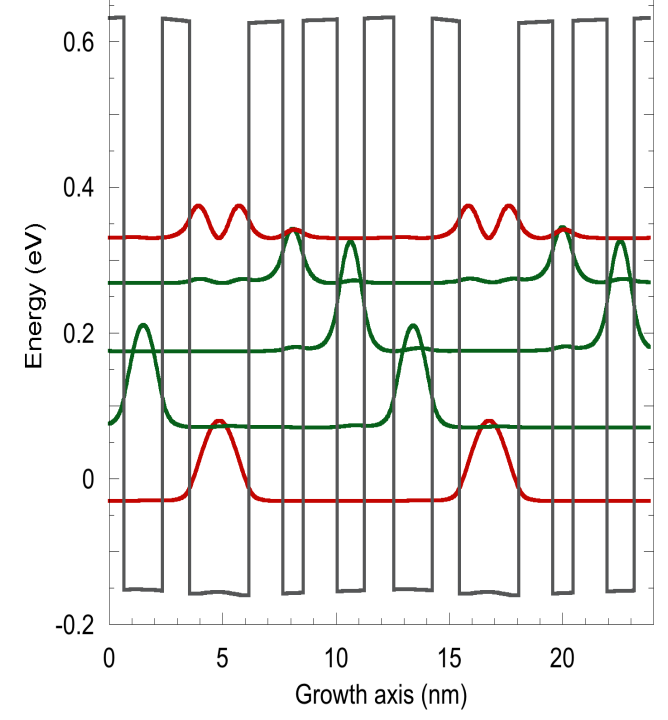

